\title{
Conventional redo biological valve replacement over 20 years: Surgical benchmarks should guide patient selection for transcatheter valve-in-valve therapy
}

John M. Stulak, MD, Vakhtang Tchantchaleishvili, MD, Richard C. Daly, MD, Mackram F. Eleid, MD, Kevin L. Greason, MD, Joseph A. Dearani, MD, Lyle D. Joyce, MD, PhD, Alberto Pochettino, MD, Hartzell V. Schaff, MD, and Simon Maltais, MD, PhD

\section{ABSTRACT}

Objectives: Although primary transcatheter valve interventions have demonstrated acceptable early- and intermediate-term outcomes, data are lacking to guide patient selection for transcatheter valve-in-valve therapy. Furthermore, very few surgical benchmarks have been established for repeat conventional biological valve replacement to refine momentum for broad application of transcatheter intervention for a degenerated bioprosthesis.

Methods: From January 1993 to July 2014, 694 patients underwent repeat biological valve replacement at our clinic. Median age at repeat operation was 71 years (range, $26-95$ years) and there were 437 men $(63 \%)$. Hypertension was present in 453 patients $(65 \%)$, diabetes in 128 patients $(18 \%)$, prior myocardial infarction in 85 patients $(12 \%)$, and prior stroke in 81 patients $(12 \%)$. Prior coronary bypass grafting was performed in 212 patients (31\%). Median left ventricular ejection fraction was $41 \%$ (range, 20-61) and New York Heart Association functional class III or IV was present in 529 patients $(76 \%)$.

Results: Biological valve re-replacement included most commonly aortic valve in 464 patients $(67 \%)$ and mitral valve in $170(24 \%)$. Concomitant coronary bypass grafting was performed in 134 patients (19\%). Mortality at 30 days occurred in 56 patients $(8 \%)$. Multivariable analysis with backward stepwise regression identified New York Heart Association functional class (per 1 increment) (hazard ratio, $2.1 ; 95 \%$ confidence interval, $1.06-4.3 ; P=.03$ ) and prior coronary bypass grafting (hazard ratio, $3.5 ; 95 \%$ confidence interval, $1.2-10.9 ; P=.03$ ) as independent predictors of early death. Patients with the combination of prior coronary bypass grafting and New York Heart Association functional class III or IV accounted for 26 out of 56 early deaths ( $46 \%$ ) and in the absence of this combination, early death in the cohort was 30 out of $694(4 \%)$. Follow-up was available in 602 out of 638 early survivors $(94 \%)$ for a median of 45 months (range, 1 month-23.4 years). Survival at 5 and 10 years was $63 \%$ and $34 \%$, respectively. For patients who died during follow-up, 2-dimensional scatter plots demonstrate durable length of post-

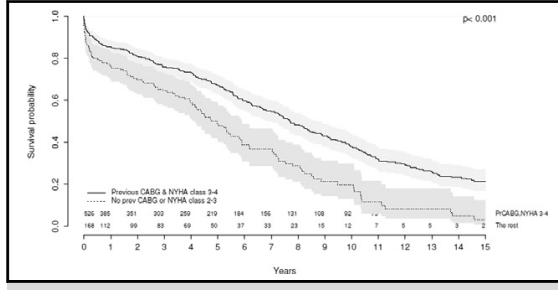

Patients with previous CABG and NYHA functional class III or IV had significantly lower survival.

\section{Central Message}

This study provides a surgical benchmark to guide patient selection for transcatheter valve-in-valve procedures because it defines high- and low-risk subgroups of patients undergoing redo biological valve replacement.

\section{Perspective}

Prior CABG and preoperative NYHA functional class III or IV was a clinical combination that imparted a higher operative risk for both aortic and mitral valve reoperation, suggesting that these patients may benefit from a lower-risk transcatheter approach. In the absence of these risk factors, conventional surgery can be performed with durable results not demonstrated by transcatheter intervention.

See Editorial Commentary page 1391.

See Editorial page 1355. operative survival (median, 5.5 years; maximum, 22 years).

Conclusions: In a large population of patients undergoing repeat biological valve replacement, prior coronary bypass grafting and advanced New York Heart Association functional class were associated with increased 30-day mortality, with the remaining population having a low 30 -day mortality of $4 \%$. This study could serve

From the Departments of Cardiovascular Surgery and Cardiovascular Diseases, Mayo Clinic and Foundation, Rochester, Minn.

Read at the 97th Annual Meeting of The American Association for Thoracic Surgery, Boston, Massachusetts, April 29-May 3, 2017.

Received for publication May 2, 2017; revisions received Feb 13, 2018; accepted for publication March 24, 2018.

\footnotetext{
Address for reprints: John M. Stulak, MD, Division of Cardiovascular Surgery, Mayo Clinic College of Medicine, 200 First St SW, Rochester, MN 55905 (E-mail: stulak.john@mayo.edu). 0022-5223/\$36.00

Copyright (C) 2018 Published by Elsevier Inc. on behalf of The American Association for Thoracic Surgery

https://doi.org/10.1016/j.jtcvs.2018.03.176
} 

Abbreviations and Acronyms
AUC = area under the curve
$\mathrm{CABG}=$ coronary artery bypass grafting
LVEF = left ventricular ejection fraction
NYHA $=$ New York Heart Association
PROM $=$ predicted risk of mortality
STS $=$ Society of Thoracic Surgeons

\section{Scanning this $\mathrm{QR}$ code will take \\ you to the supplemental table. \\ To view the AATS Annual \\ Meeting Webcast, see the \\ URL next to the webcast \\ thumbnail.}

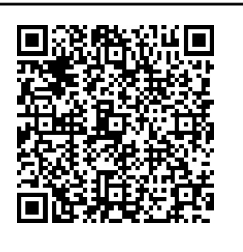

as a surgical benchmark to guide patient selection for transcatheter valve-in-valve technology rather than employing a broader application of these techniques to those who may otherwise have low early risk of mortality and durable long-term survival after conventional valve surgery. (J Thorac Cardiovasc Surg 2018;156:1380-90)

Increasing trends in the use of biological implants at the time of surgery to correct valvular heart disease ${ }^{1}$ will inevitably lead to an escalating burden of structural deterioration due to the limited lifespan of these prostheses. Conventional redo surgery currently represents the standard of care, but reoperation for a degenerated valve prosthesis can carry a high risk of morbidity and mortality, especially in elderly patients or those with significant comorbidities. ${ }^{2,3}$ Although a large body of literature exists supporting transcatheter aortic valve replacement as an alternative to surgery for patients at high operative risk, ${ }^{4-8}$ only a modest, but growing, collection of reports exist demonstrating early observations of applying this approach to treat a degenerated bioprosthesis. ${ }^{9-13}$ Analysis of a larger, multinational valve-in-valve registry has highlighted important issues to be considered before widespread application of this technology to this new patient population is appropriate. ${ }^{14,15}$

Although there are emerging reports on comparisons of transcatheter approaches with redo conventional valve re-replacement, ${ }^{16-18}$ there are very few data analyzing surgical series to identify patients who are at particular risk for conventional surgery in whom a selective transcatheter approach might be optimal. We sought to analyze patients undergoing conventional redo aortic or mitral biological valve replacement to identify risk factors, evaluate early and late survival, and assess current ability to assess risk preoperatively to also identify patients who may otherwise have low early risk of and durable long-term survival after conventional redo biological valve surgery.

\section{PATIENTS AND METHODS}

The Mayo Foundation Institutional Review Board approved this study, and all patients or their families gave written informed consent.

\section{Patients}

From January 1993 to July 2014, 694 patients underwent repeat biological valve replacement at our clinic. Median age at repeat operation was 71 years (range, 26-95 years) and there were 437 men (63\%). Patient cohorts included most commonly redo aortic valve replacement in 464 patients $(67 \%)$ and redo mitral valve replacement in 170 patients $(24 \%)$. Patients requiring redo valve replacement due to endocarditis or under urgent/emergent conditions were excluded from this analysis to duplicate the current clinical scenario in which patients are considered for percutaneous valve insertion.

\section{Preoperative Clinical Characteristics}

Preoperative clinical characteristics for the entire cohort are presented in Table 1 and Table E1 and most commonly included coronary artery disease in 477 patients $(69 \%)$, hypertension in 453 patients $(65 \%)$, and chronic lung disease in 410 patients $(59 \%)$. Median preoperative left ventricular ejection fraction (LVEF) was $41 \%$ (range, 20\%-61\%) and New York Heart Association (NYHA) functional class III or IV was present in 529 patients $(76 \%)$. Median preoperative Society of Thoracic Surgeons (STS) predicted risk of mortality was 5.5\% (range, 0.8\%-62\%).

Preoperative clinical characteristics stratified for the aortic and mitral valve cohorts are presented in Table 2 . For the aortic valve cohort, median preoperative LVEF was $41 \%$ (range, $20 \%-61 \%$ ) and NYHA functional class III or IV was present in 320 patients $(69 \%)$. Median preoperative STS predicted risk of mortality for this cohort was $4.9 \%$ (range, $0.8 \%-62 \%$ ). For the mitral valve cohort, median preoperative LVEF was $60 \%$ (range, $25 \%-86 \%$ ) and NYHA functional class III or IV was present in 155 patients ( $91 \%$ ). Median preoperative STS predicted risk of mortality for this cohort was $7.5 \%$ (range $0.9 \%-58 \%$ ).

\section{Statistical Analysis}

Demographic and other patient-related data were obtained from Mayo Clinic medical records and our prospective clinical database. Follow-up information was obtained from subsequent clinic visits, written correspondence from local physicians, and mailed questionnaires to patients or families. Continuous data were expressed as median value with a range due to significant skewness. Categorical variables were expressed as percentages. Multivariable logistic regression analysis was performed to identify predictors of 30-day mortality, whereas multivariable Cox proportional hazards method was used for time-to-event analysis to identify predictors of late survival. Multivariable models were developed using backward stepwise regression based on the lowest value of Akaike information criterion. ${ }^{19}$ Input to the stepwise regression consisted of patient demographic variables, comorbidities, and previous surgery. Survival was determined using Kaplan-Meier analysis and survival comparisons between 2 samples were assessed with log-rank test. Receiver operating characteristic analysis will be used to derive areas under the curve (AUC) for assessing the discriminatory power of the STS risk models in predicting early postoperative mortality. We then applied the Hosmer-Lemeshow test to the entire data and both subsets (aortic and mitral valve) to test calibration of the STS risk model. Early operative mortality was defined as death occurring within 30 days of operation or any time during the index hospitalization. R statistical software, version 3.2.3 (R Foundation for Statistical Computing, Vienna, Austria) was used for data analysis and visualization. 
TABLE 1. Preoperative clinical characteristics present in the entire cohort

\begin{tabular}{lc}
\hline \multicolumn{1}{c}{ Variable } & Result \\
\hline Age (y) & $72(27-95)$ \\
Coronary artery disease & $477(69)$ \\
Hypertension & $453(65)$ \\
Chronic lung disease & $410(59)$ \\
\hline Prior coronary artery bypass grafting & $212(31)$ \\
Cerebrovascular disease & $150(22)$ \\
Diabetes & $128(18)$ \\
Prior stroke & $81(12)$ \\
\hline Peripheral vascular disease & $71(10)$ \\
Prior myocardial infarction & $51(7)$ \\
Immunosuppression & $41(6)$ \\
\hline
\end{tabular}

Values are presented as median (range) or $\mathrm{n}(\%)$.

\section{RESULTS}

\section{Operative Data}

All patients underwent redo biological valve replacement, including redo aortic valve replacement in 464 patients $(67 \%)$ and redo mitral valve replacement in 170 patients $(24 \%)$ most commonly.

For the entire cohort, median cardiopulmonary bypass time was 125 minutes (range, 32-674 minutes) and median aortic crossclamp time was 85 minutes (range, 20-416 minutes). Concomitant coronary artery bypass grafting (CABG) was performed in 134 patients (19\%). Immediate postoperative intra-aortic balloon pump was required in 87 patients $(13 \%)$ and extracorporeal membrane oxygenation was required in 6 patients $(0.9 \%)$. Mediastinal re-exploration for bleeding was required in 42 patients $(6 \%)$.

For the redo aortic valve cohort, median cardiopulmonary bypass time was 131 minutes (range, 32-674 minutes) and median aortic crossclamp time was 90 minutes (range, 19-416 minutes). Concomitant CABG was performed in

TABLE 2. Preoperative clinical characteristics present in the aortic and mitral valve cohorts

\begin{tabular}{lcc}
\hline \multicolumn{1}{c}{ Variable } & $\begin{array}{c}\text { Aortic valve } \\
(\mathbf{n}=\mathbf{4 6 4})\end{array}$ & $\begin{array}{c}\text { Mitral valve } \\
(\mathbf{n}=\mathbf{1 7 0})\end{array}$ \\
\hline Age $(\mathrm{y})$ & $72(27-95)$ & $70(28-89)$ \\
Men & $341(73)$ & $69(41)$ \\
\hline Diabetes & $82(18)$ & $37(22)$ \\
Hypertension & $317(68)$ & $106(62)$ \\
Chronic lung disease & $293(63)$ & $84(49)$ \\
Immunosuppression & $24(5)$ & $12(7)$ \\
\hline Peripheral vascular disease & $49(11)$ & $13(7)$ \\
Cerebrovascular disease & $81(17)$ & $49(29)$ \\
\hline Prior coronary artery bypass grafting & $154(33)$ & $46(27)$ \\
Prior stroke & $43(9)$ & $26(15)$ \\
\hline Prior myocardial infarction & $35(8)$ & $15(9)$ \\
\hline
\end{tabular}

Values are presented as median (range) or $\mathrm{n}(\%)$.
TABLE 3. Early nonfatal morbidity observed in the entire cohort

\begin{tabular}{lc}
\hline \multicolumn{1}{c}{ Variable } & n (\%) \\
\hline Prolonged mechanical ventilation & $164(24)$ \\
Renal insufficiency & $77(11)$ \\
Renal failure requiring dialysis & $49(7)$ \\
New permanent pacemaker & $46(6.6)$ \\
Wound infection & $26(3.7)$ \\
Any neurologic event (stroke + transient ischemic attack) & $11(1.6)$ \\
\hline
\end{tabular}

105 patients $(23 \%)$. Immediate postoperative intra-aortic balloon pump was required in 61 patients $(13 \%)$ and extracorporeal membrane oxygenation was required in 5 patients $(1.1 \%)$. Mediastinal re-exploration for bleeding was required in 32 patients $(7 \%)$.

For the redo mitral valve cohort, median cardiopulmonary bypass time was 111 minutes (range, 42-302 minutes) and median aortic crossclamp time was 70 minutes (range, 20-178 minutes). Concomitant CABG was performed in 22 patients $(13 \%)$. Immediate postoperative intra-aortic balloon pump was required in 22 patients (13\%). Mediastinal re-exploration for bleeding was required in 7 patients $(4 \%)$.

\section{Early Outcomes}

Early nonfatal morbidity for the entire cohort is presented in Table 3 and most commonly included prolonged mechanical ventilation in 164 patients $(24 \%)$ and renal insufficiency in 77 patients (11\%). Early nonfatal morbidity stratified according to the redo aortic and redo mitral valve cohorts is presented in Table 4.

For the entire cohort, early death occurred in 56 patients $(8 \%)$. Multivariable analysis with backward stepwise regression identified NYHA functional class (per 1 increment) (hazard ratio [HR], 2.1; 95\% confidence interval $[\mathrm{CI}], 1.06-4.3 ; P=.03$ ) and prior CABG (hazard ratio, 3.5; $95 \% \mathrm{CI}, 1.2-10.9 ; P=.03$ ) as independent predictors of early death. Patients with the combination of prior CABG and NYHA functional class III or IV accounted for 26 out of 56 early deaths $(46 \%)$ for an early mortality rate of 26 out of $168(15.5 \%)$ and in the absence of this preoperative combination, early death in the cohort was 30 out of $526(5.7 \%)$.

TABLE 4. Early nonfatal morbidity observed in the aortic and mitral valve cohorts

\begin{tabular}{lcc}
\hline \multicolumn{1}{c}{ Variable } & $\begin{array}{c}\text { Aortic valve } \\
(\mathbf{n}=\mathbf{4 6 4})\end{array}$ & $\begin{array}{c}\text { Mitral valve } \\
(\mathbf{n}=\mathbf{1 7 0})\end{array}$ \\
\hline Prolonged mechanical ventilation & $108(23)$ & $42(25)$ \\
Renal insufficiency & $52(11)$ & $15(9)$ \\
Renal failure requiring dialysis & $33(7)$ & $9(5)$ \\
New permanent pacemaker & $30(6.4)$ & $15(9)$ \\
Wound infection & $21(4)$ & $4(2)$ \\
$\begin{array}{l}\text { Any neurologic event } \\
\text { (stroke }+ \text { transient ischemic attack) }\end{array}$ & $8(1.7)$ & $2(1.2)$ \\
\hline
\end{tabular}




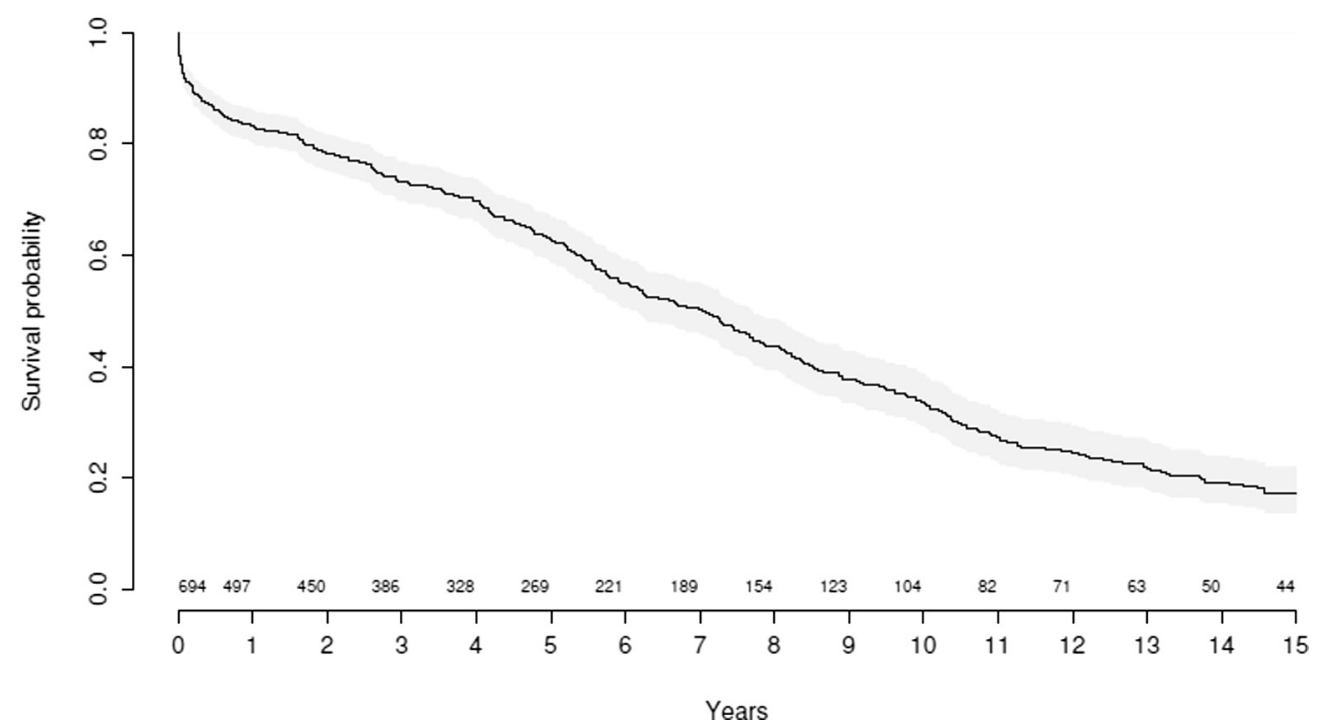

FIGURE 1. Late survival for all patients undergoing conventional redo biological valve replacement.

\section{Late Outcomes}

Follow-up was available in 602 out of 638 early survivors $(94 \%)$ for a median of 45 months (range, 1 month-23.4 years). For the entire cohort, survival at 5 and 10 years was $63 \%$ and $34 \%$, respectively (Figure 1). For the redo aortic valve cohort, survival at 5 and 10 years was $63 \%$ and $34 \%$, respectively, and for the redo mitral valve cohort, survival at 5 and 10 years was $61 \%$ and $32 \%$, respectively. For patients who were deceased at last follow-up, 2-dimensional scatter plots demonstrates varying length of postoperative survival (median, 5.5 years; maximum, 22 years) to visually demonstrate potential for durable outcome (Figure 2). Multivariable predictors independently associated with late death are presented in Table 5.

To identify a patient population at particularly high risk for redo conventional valve replacement and who may benefit most from an alternate approach, we analyzed survival for the combination of patients identified in multivariable analysis as having inferior early survival (prior CABG and NYHA functional class III or IV). In the entire cohort, patients with previous CABG and preoperative NYHA functional class III or IV had significantly lower survival compared with patients without this preoperative combination (Figure 3). When analyzed within the aortic and mitral valve subset, this combination of preoperative risk factors significantly influenced late survival in a similarly adverse manner (Figures 4 and 5, respectively). To analyze outcomes from a temporal standpoint, we examined survival for index operations performed in the 1990s, 2000s, and after 2010. This is presented in Figure 6 and demonstrates that outcomes were significantly different according to decade of surgery performed $(P<.01)$; survival of patients of patients was significantly higher when operation was performed in the 1990s.

Preoperative risk stratification was undertaken utilizing the STS predicted risk of mortality (PROM) calculator. Median preoperative STS PROM was $5.5 \%$ (range, $0.8 \%-62 \%$ ) for the entire cohort, $4.9 \%$ (range, $0.8 \%-62 \%$ ) for the redo aortic valve cohort, and $7.5 \%$ (range, $0.9 \%-58 \%$ ) for the redo mitral valve cohort. Utilizing receiver operating characteristic analysis to assess the discriminatory power of the STS PROM, we observed a good performance for risk prediction in the redo aortic

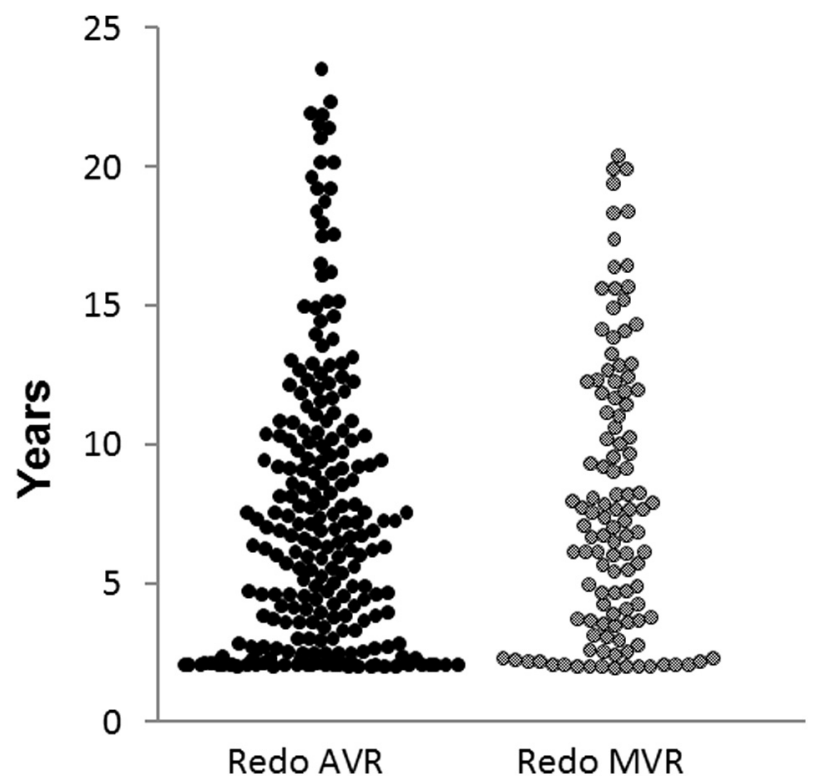

FIGURE 2. For patients who were deceased at last follow-up, 2-dimensional scatter plots demonstrate varying length of postoperative survival. AVR, Aortic valve replacement; $M V R$, mitral valve replacement. 
TABLE 5. Multivariate predictors associated with late survival

\begin{tabular}{|c|c|c|}
\hline Variable & $\begin{array}{c}\text { Hazard ratio } \\
\text { (95\% Confidence } \\
\text { interval) }\end{array}$ & $\begin{array}{c}P \\
\text { value }\end{array}$ \\
\hline \multicolumn{3}{|l|}{ Entire cohort } \\
\hline Age (per decade) & $1.03(1.02-1.04)$ & $<.01$ \\
\hline $\begin{array}{l}\text { New York Heart association } \\
\text { functional class }\end{array}$ & $1.18(1.02-1.37)$ & .02 \\
\hline Postoperative creatinine level & $1.26(1.14-1.39)$ & $<.01$ \\
\hline Peripheral vascular disease & $1.48(1.01-2.17)$ & .04 \\
\hline \multicolumn{3}{|l|}{ Redo aortic valve replacement cohort } \\
\hline Age (per decade) & $1.03(1.02-1.05)$ & $<.01$ \\
\hline $\begin{array}{l}\text { New York Heart Association } \\
\text { functional class }\end{array}$ & $1.17(1.00-1.38)$ & .05 \\
\hline Postoperative creatinine level & $1.27(1.13-1.42)$ & $<.01$ \\
\hline Prior coronary artery bypass grafting & $1.49(1.09-2.03)$ & .01 \\
\hline \multicolumn{3}{|l|}{ Redo mitral valve replacement cohort } \\
\hline Age (per decade) & $1.04(1.01-1.07)$ & .01 \\
\hline $\begin{array}{l}\text { New York Heart Association } \\
\text { functional class }\end{array}$ & $1.63(1.00-2.67)$ & .05 \\
\hline Postoperative creatinine level & $2.31(1.33-4.01)$ & $<.01$ \\
\hline Peripheral vascular disease & $2.69(1.04-6.99)$ & .04 \\
\hline
\end{tabular}

valve cohort with an AUC of $88.3 \%$ (83.4\%-93.2\%) (Figure 7) and poor performance in the redo mitral valve cohort with an AUC of $60.7 \% \quad(25.5 \%-95.9 \%)$ (Figure 8). We then applied Hosmer-Lemeshow test to the entire data and both subsets (aortic and mitral valve surgeries) and found poor calibration for each of them
$(P=.009$ for the aortic valve surgery subset, $P=.014$ for the mitral valve surgery subset, and $P=.001$ for the entire data).

\section{DISCUSSION}

This large analysis of patients undergoing conventional redo biological valve replacement highlights numerous important data that are important in helping to clarify which patient population may be best served with either a transcatheter approach or conventional surgery. In the entire cohort, early mortality was similar to that of new reports examining transcatheter and conventional approaches to a degenerated bioprosthesis, but we observed particularly high early mortality in patients with preoperative NYHA functional class III or IV and those who have undergone prior CABG. Indeed, almost half of all early deaths were in patients with this combination of preoperative risk factors. When survival was analyzed comparing patients with and without this combination of risk factors, we observed a significantly improved early and late survival in the absence of these clinical factors. Perhaps in these patients, a surgical approach should be favored as a durable, long-term outcome with low early risk can be anticipated. Furthermore, we observed a low rate of any neurologic events and need for permanent pacemaker, which have varied in the early reports of valve-in-valve techniques. ${ }^{14-18}$

Identifying the optimal patient population is clearly critical to the long-term success and efficacy of both approaches and early reports on transcatheter valve-in-valve

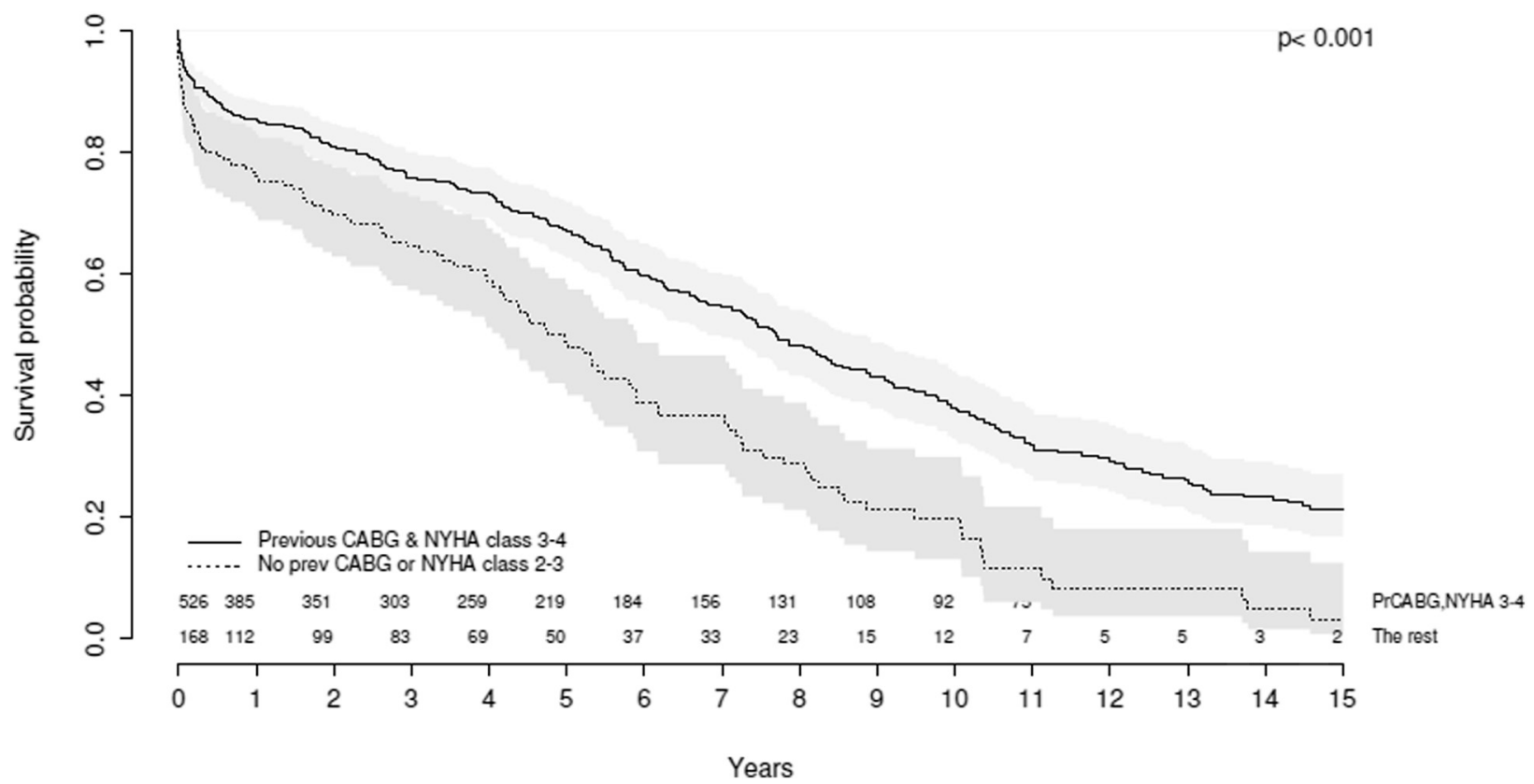

FIGURE 3. For the entire cohort, late survival for patients with preoperative New York Heart Association (NYHA) functional class III or IV and prior coronary artery bypass grafting ( $p r C A B G)$ is shown compared with patients without this preoperative combination of clinical risk factors. Patients with previous (prev) CABG and preoperative NYHA functional class III or IV had significantly lower survival compared with patients without this preoperative combination of clinical risk factors. $C A B G$, Coronary artery bypass grafting. 


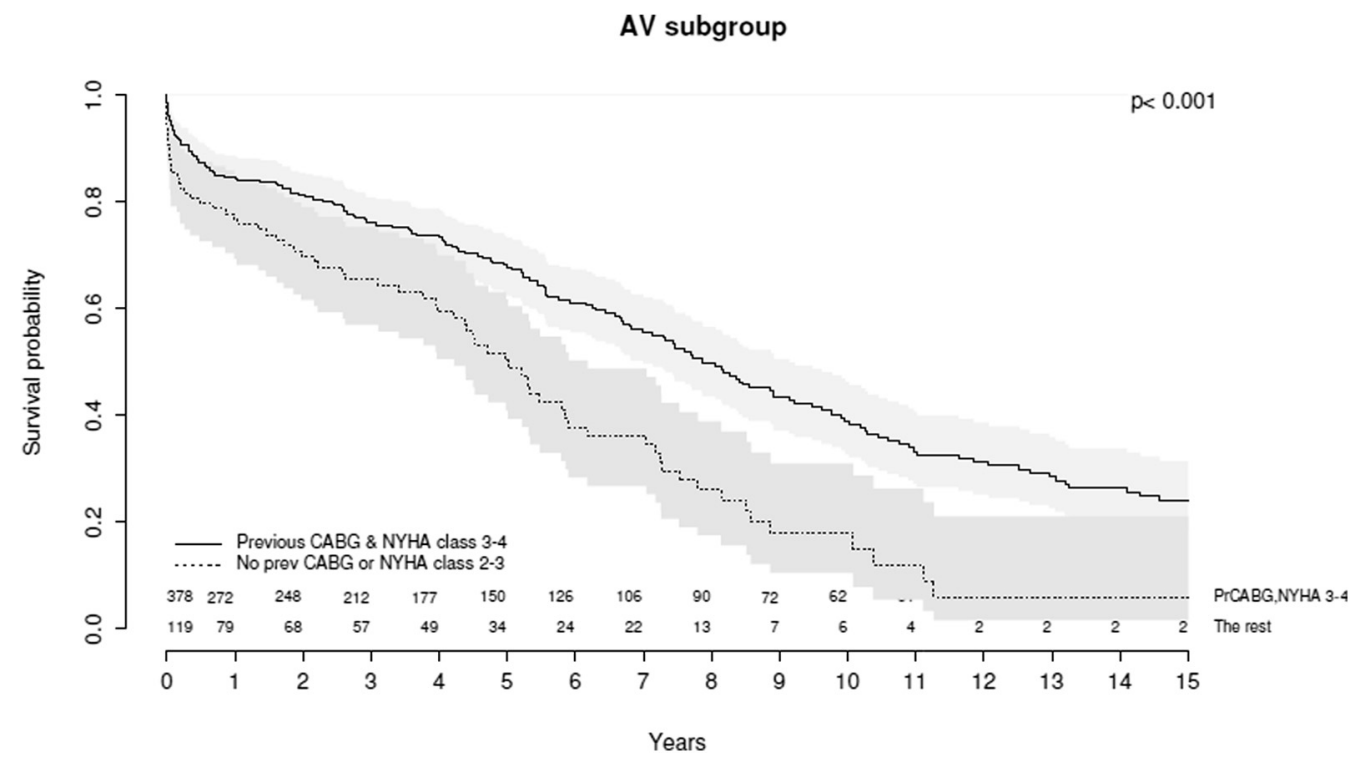

FIGURE 4. For the redo aortic valve $(A V)$ cohort, late survival for patients with preoperative New York Heart Association (NYHA) functional class III or IV and prior coronary artery bypass grafting $(\mathrm{prCABG})$ is shown compared with patients without this preoperative combination of clinical risk factors. Patients with previous CABG and preoperative NYHA functional class III or IV had significantly lower survival compared with patients without this preoperative combination of clinical risk factors. $C A B G$, Coronary artery bypass grafting.

still lack strong data on long-term durability of these prostheses especially in the face of higher gradients and lower effective orifice areas observed early after implantation. ${ }^{14,15}$ Although we do not report long-term durability of the surgically implanted prostheses, we did observe good long-term survival, which is higher than that reported to date in the early valve-in-valve experience. ${ }^{14,15}$
For those who died during follow-up, we observed a majority had favorable long-term survival after conventional surgery (Figure 2). Registry data have similarly evaluated risk factors placing patients at higher periprocedural risk and lower long-term survival after valve-in-valve procedure, with smaller surgical prosthesis and baseline valvular stenosis being associated with poor

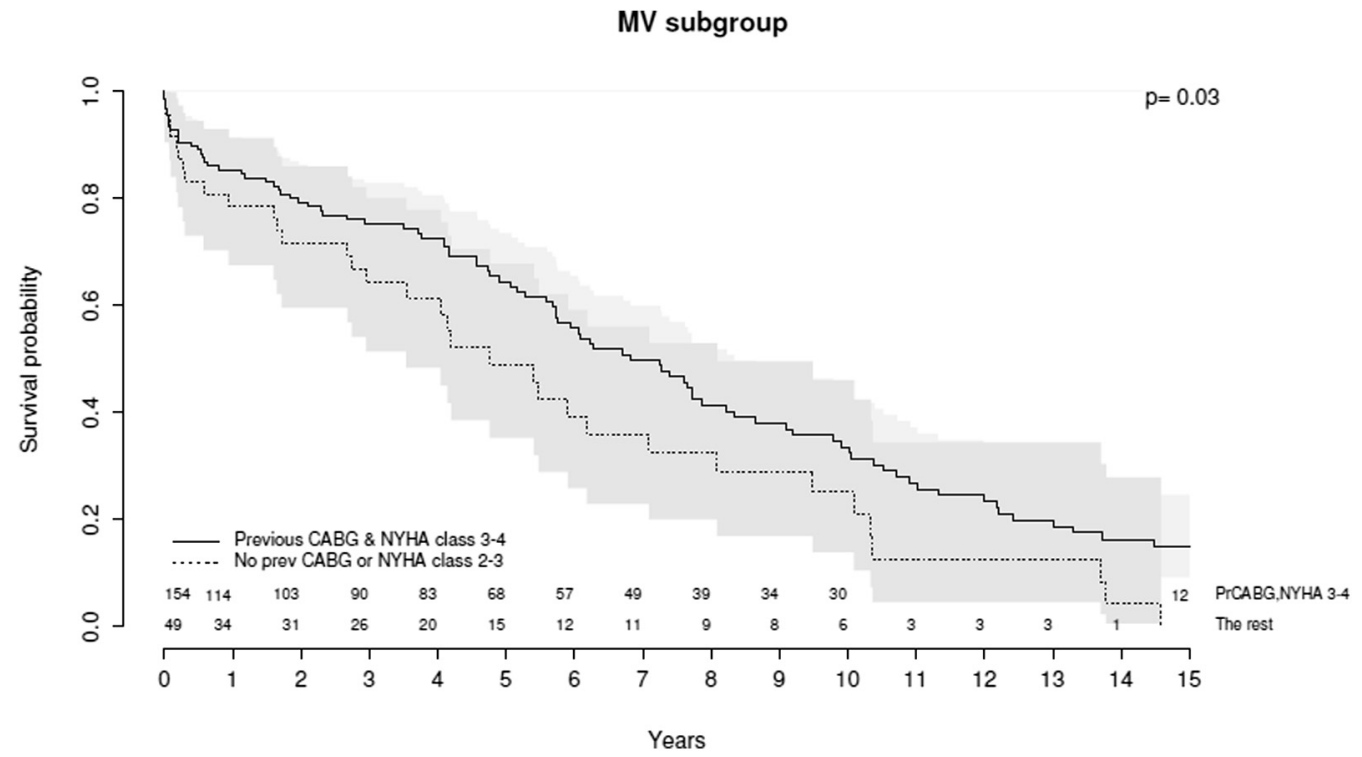

FIGURE 5. For the redo mitral valve ( $M V$ ) cohort, late survival for patients with preoperative New York Heart Association (NYHA) functional class III or IV and prior coronary artery bypass grafting $(p r C A B G)$ is shown compared with patients without this preoperative combination of clinical risk factors. Patients with previous CABG and preoperative NYHA functional class III or IV had significantly lower survival compared with patients without this preoperative combination of clinical risk factors. $C A B G$, Coronary artery bypass grafting. 


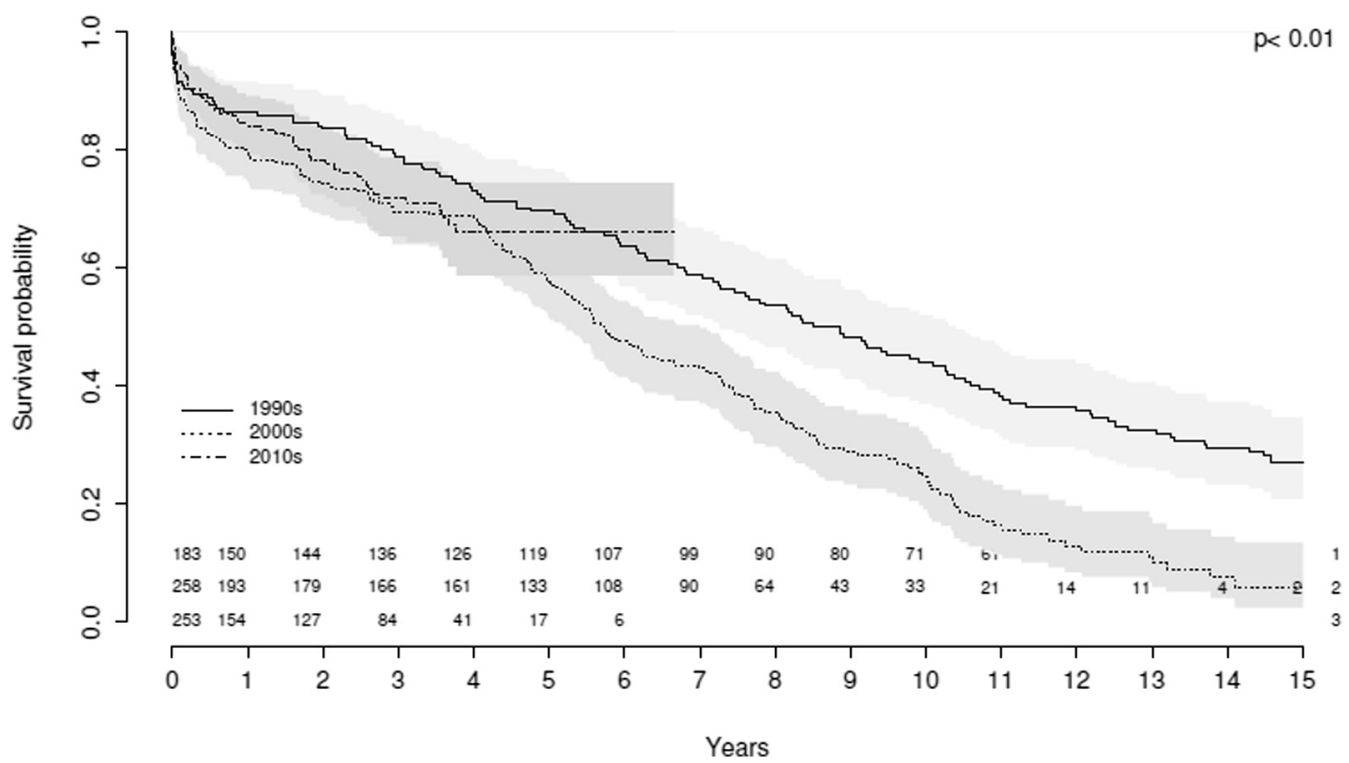

FIGURE 6. Survival according to era of surgery is presented. There is a significant difference between survival for patients having surgery during 1990 s compared with more contemporary era $(P<.01)$.

outcomes. These factors also represent a patient subgroup in which conventional redo surgery should be considered as the preferred approach when feasible.

The evaluation of risk for patients undergoing either transcatheter or conventional valvular procedures has relied most heavily on the STS PROM calculator. This has been

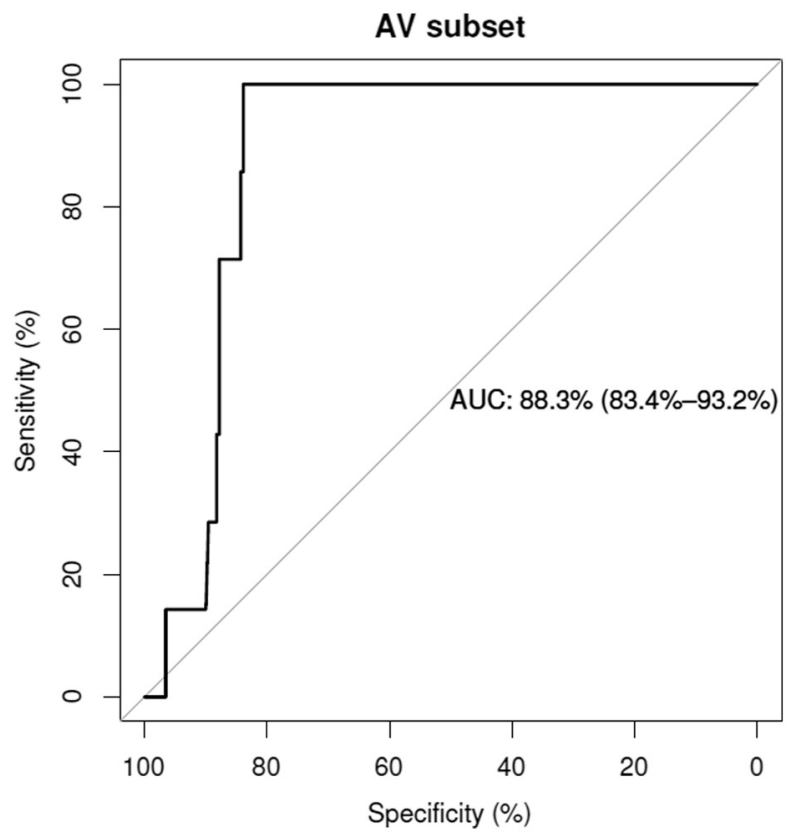

FIGURE 7. Receiver operating characteristic analysis to assess the discriminatory power of the Society of Thoracic Surgeons predicted risk of mortality is shown for the redo aortic valve $(A V)$ cohort. AUC, Area under the curve. used as the preferred method to define the patient subgroups studied in the landmark trials establishing transcatheter approaches in native valve disease. ${ }^{4-7}$ We evaluated the performance of this predictive risk model in this cohort and found disparate performance of the STS risk model for the aortic and mitral valve cohort. Predictive ability

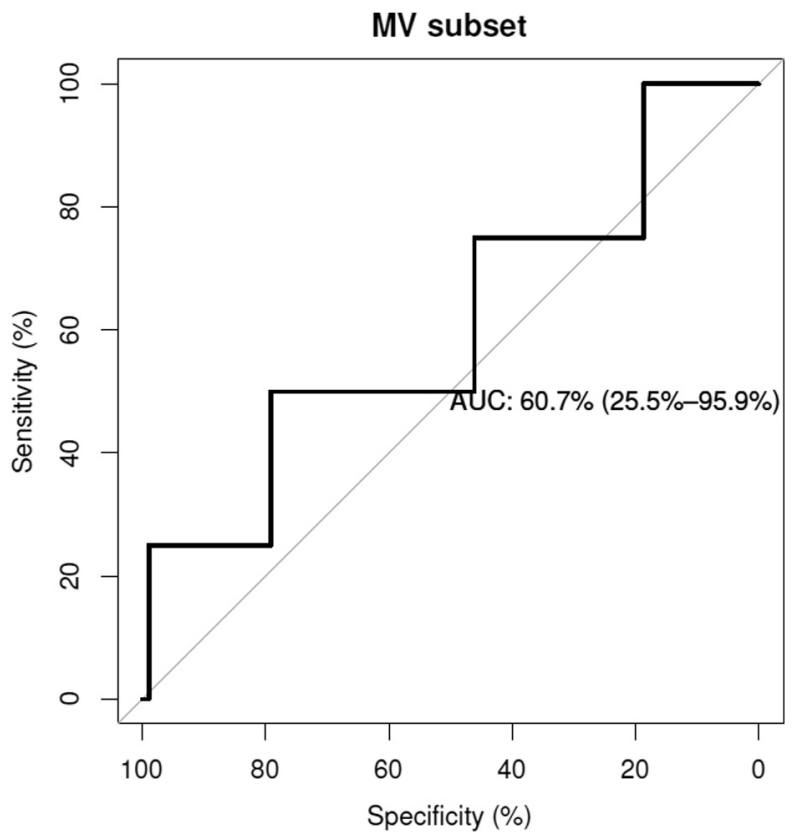

FIGURE 8. Receiver operating characteristic analysis to assess the discriminatory power of the Society of Thoracic Surgeons predicted risk of mortality is shown for the redo mitral valve $(M V)$ cohort. AUC, Area under the curve. 
was much higher for the redo aortic valve cohort with an AUC of $88.3 \%$ compared with the redo mitral valve cohort in which the AUC was $60.7 \%$. This is an important concept to consider when entertaining which approach is optimal for a patient with a degenerated bioprosthesis. Although this may be the best tool available to assess the risk profile for a patient being evaluated for treatment, its inconsistent ability to accurately predict risk in these patients needs to be factored into the decision making. Sole reliance on a high STS risk as the driver to consider the transcatheter approach may unnecessarily restrict offering conventional surgery to a patient who may otherwise benefit from that approach. A more holistic approach as recommended in the American College of Cardiology/American Heart Association guidelines for the management of patients with valvular heart disease incorporating not only STS risk score, but the presence of frailty criteria, procedure-specific impediments, and major organ system dysfunction is likely to improve the accuracy of patient risk assessment. ${ }^{20}$ Furthermore, the timing of referral for surgery is a major issue and an issue that was apparent in this study with such a high proportion of patients coming to surgery with NYHA functional class III or IV symptoms. This would perhaps indicate that referral for surgery was delayed favoring ongoing medical therapy. Temporally related outcomes in this study demonstrate that surgery performed in the 1990s was associated with better outcomes, and one could postulate that there was earlier referral for surgery compared with the more current era.

It is important to note that recent comparisons of transcatheter and conventional surgical approaches demonstrated similar early outcomes, ${ }^{16-18}$ which further validates that conventional surgical approaches are possible in these challenging patients. It would be surmised that a less- invasive, transcatheter approach would impart a lower early risk because of the avoidance of redo sternotomy, cardiopulmonary bypass, and cardioplegic arrest. It should be noted that these outcomes are being reported for all comers without stratification of risk. Herein lays the value in this series in that the establishment of a benchmark from which to anticipate outcomes is of the utmost importance.

While reviewing early outcomes and optimizing exposure to risk, consideration of durability and long-term survival needs to be considered. Because of the shorter time in existence for transcatheter approaches, these data are not available at this time. However, we demonstrate risk factors associated with adverse late survival, which includes age, NYHA functional class, postoperative renal function, peripheral vascular disease, and prior CABG. These risk factors are consistently identified as being influential on outcomes following cardiac surgery and it is not surprising that these clinical factors should also be taken into consideration before a decision on the optimal approach is defined. It is natural that the focus on new technology is on early risk and procedural success, but the data reported in this study support that early outcomes should not be the sole factor considered. Herein also lays the nature of the challenge selecting the optimal approach in these complex patients.

As the history and progression of transcatheter approaches have demonstrated, these complex decisions on treatment options offered to patients should take place in a multidisciplinary manner. Furthermore, this is the expectation not only from a guidelines standpoint, but also the government payer. In this manner, consideration of all patient factors is more often to be evaluated and a balance between the natural bias of individual practitioners can be attained.

\section{Limitations}

This is a retrospective review of a prospectively collected database from a single institution. Very modest and traditional statistical methods were applied to establish patient populations at high and low risk for the surgical procedure studied. No comparisons to transcatheter approaches were undertaken in this series. Modes of death and functional outcomes at last follow-up were not available to an appreciable extent. Although this was not the intent of the study, this is an important limitation to be acknowledged. Upon analyzing the missing data, we concluded that they were missing completely at random and no pattern to missing data was identified. Where possible, the missing data from the STS database were recovered from electronic medical records. With very little missing data after this maneuver, if very few were missing for a certain characteristic, then listwise deletion was employed when the sample size was not threatened. High censorship in this long-term data could have skewed results in this cohort. Patients lost to follow-up were most commonly those who lived a long distance from our institution and who did not return for formal visit. However, a concerted effort was made to correspond with the local providers to ascertain clinical status. Another population of patients who are often lost to follow-up are those who are doing well and simply do not choose to return for formal follow-up. Although no assumptions are made with these patients or data, if this were primarily the case, and their outcomes were censored, this would yield our reported outcomes as underappreciating the success of these procedures.

\section{CONCLUSIONS}

We analyzed all patients undergoing redo conventional biological valve replacement to identify subsets of patients at high risk who may benefit from a transcatheter approach and also patients at lower risk who otherwise 
may benefit from a conventional operation. Prior CABG and preoperative NYHA functional class III or IV was a clinical combination that imparted a higher operative risk for both aortic and mitral valve reoperation suggesting that these patients may benefit from a lower-risk transcatheter approach. STS risk model had only fair discriminatory power in the mitral valve subset and good discriminatory power for predicting early risk in these patients, so consideration of other factors such as predictors of long-term survival may assist in clinical decision making as to the optimal approach in these challenging patients.

\section{Webcast}

You can watch a Webcast of this AATS meeting presentation by going to: https://aats.blob.core.windows. net/media/17AM/2017-05-01/BallroomABC/05-01-17_ BallroomABC_1644_Stulak.mp4.

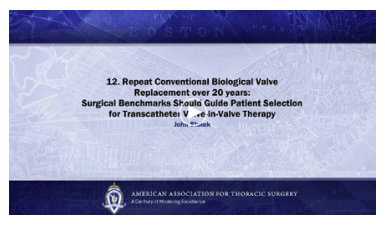

\section{Conflicts of Interest Statement}

Authors have nothing to disclose with regard to commercial support.

\section{References}

1. Brown JM, O'Brien SM, Wu C, Sikora JA, Griffith BP, Gammie JS. Isolated aortic valve replacement in North America comprising 108,687 patients in 10 years: changes in risks, valve types and outcomes in the Society of Thoracic Surgeons national database. J Thorac Cardiovasc Surg. 2009;137:82-90.

2. Maganti M, Rao V, Armstrong S, Feindel CM, Scully HE, David TE. Redo valvular surgery in elderly patients. Ann Thorac Surg. 2009;87:521-5.

3. Kirsch M, Nakashima K, Kubota S, Houel R, Hillion ML, Loisance D. The risk of reoperative heart valve procedures in octogenarian patients. J Heart Valve Dis. 2004;13:991-6.

4. Leon MB, Smith CR, Mack M, Miller DC, Moses JW, Svensson LG, et al. Transcatheter aortic valve implantation for aortic stenosis in patients who cannot undergo surgery. N Engl J Med. 2010;363:1597-607.

5. Smith CR, Leon MB, Mack M, Miller DC, Moses JW, Svensson LG, et al Transcatheter vs. surgical aortic valve replacement in high risk patients. N Engl J Med. 2011:364:2187-98.

6. Kodali SK, Williams MR, Smith CR, Svensson LG, Webb JG, Makkar RR, et al. Two-year outcomes after transcatheter or surgical aortic valve replacement. N Engl J Med. 2012;366:1686-95.

7. Makkar RR, Fontana H, Jilahawi H, Kapadia S, Pichard AD, Douglas PS, et al Transcatheter aortic valve replacement for inoperable severe aortic stenosis. N Engl J Med. 2012;366:1696-704.

8. Gilard M, Eltchaninoff H, Lung B, Donzeau-Gouge P, Chevreul K, Fajadet J, et al. Registry of transcatheter aortic valve implantation in high risk patients. N Engl J Med. 2012;366:1705-15.

9. Webb JG, Wood DA, Ye J, Gurvitch R, Masson JB, Rodes-Cabau J, et al. Transcatheter valve-in-valve implantation for failed bioprosthetic heart valves. Circulation. 2010;121:1848-57.

10. Khwaja MZ, Haworth P, Ghuran A, Lee L, de Beider A, Hutchinson N, et al. Transcatheter aortic valve implantation for stenosed and regurgitant aortic valve bioprostheses CoreValve for failed bioprosthetic aortic valve replacements. J Am Coll Cardiol. 2010;55:97-101.
11. Piazza N, Bleiziffer S, Brockmann G, Hendrick R, Deutsch MA, Opitz A, et al. Transcatheter aortic valve implantation for failing surgical aortic bioprosthetic valve: from concept to clinical application and evaluation (part 2). JACC Cardiovasc Interv. 2011;4:733-42.

12. Bedgoni F, Laudisa ML, Pizzocri S, Tamburino C, Ussia GP, Petronio AS, et al. Transcatheter valve-in-valve implantation using CoreValve revalving system for failed surgical aortic bioprostheses. JACC Cardiovasc Interv. 2011;4:1228-34.

13. Eggebrecht H, Schafer U, Treede H, Boekstegers P, Babin-Ebell J, Ferrari M, et al. Valve-in-valve transcatheter aortic valve implantation for degenerated bioprosthetic heart valves. JACC Cardiovasc Interv. 2011;4:1218-27.

14. Dvir D, Webb J, Brecker S, Bleiziffer S, Hildick-Smith D, Colombo A, et al. Transcatheter aortic valve replacement for degenerative bioprosthetic surgical valves: results from the global valve-in-valve registry. Circulation. 2012;126: 2335-44.

15. Dvir D, Webb JG, Bleiziffer S, Pasic M, Waksman R, Kodali S, et al. Transcatheter aortic valve implantation in failed bioprosthetic surgical valves. JAMA. 2014;312:162-70.

16. Silaschi M, Wendler O, Seiffert M, Castro L, Lubos E, Schirmer J, et al. Transcatheter valve-in-valve implantation versus redo surgical aortic valve replacement in patients with failed aortic bioprostheses. Interact Cardiovasc Thorac Surg. 2017;24:63-70.

17. Phan K, Zhao DF, Wang N, Huo YR, Di Eusanio M, Yan TD. Transcatheter valve-in-valve implantation versus reoperative conventional aortic valve replacement: a systematic review. J Thorac Dis. 2016;8:E83-93.

18. Toumousoglou C, Rao V, Lalos S, Dougenis D. What is the best approach in a patient with a failed aortic bioprosthetic valve: transcatheter aortic valve replacement or redo aortic valve replacement? Interactiv Cardiovasc Thorac Surg. 2015;20:837-43.

19. Akaike H. A new look at the statistical model identification. IEEE Trans Autom Control. 1974;19:716-23.

20. Nishimura RA, Otto CM, Bonow RO, Carabello BA, Erwin JP III, Guyton RA, et al. 2014 AHA/ACC guidelines for the management of patients with valvular heart disease: a report of the American College of Cardiology/American Heart Association task force on practice guidelines. Circulation. 2014;129:e521-643.

Key Words: valve-in-valve, valve replacement, bioprosthesis

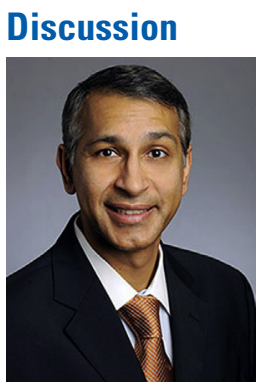

Dr Vinod H. Thourani (Washington, $D C$ ). I would like to thank the Association for the opportunity to discuss this excellent article by Dr Stulak and his colleagues from Mayo Clinic, and I would like to thank John for sending me the article and slides in advance.

The authors evaluated 694 patients undergoing redo surgery for biologic valves with a Society of Thoracic Surgeons predicted risk of mortality of 7.5, just at the cusp of high risk. They noted a 5-year survival of 63\% and $34 \%$, and it was similar to those undergoing redo aortic valve repair (AVR) and mitral valve repair. Specifically, they noted that prior coronary artery bypass grafting or New York Heart Association functional class III or IV lead to significantly higher early and late mortality. They note-and I agree - that they have had excellent results in redo valve surgery.

I have a few questions. It seems that a major limitation of the current study is a lack of echocardiographic analysis. One of the major limitations of transcatheter AVR valve-in-valve, as was just mentioned, is that it results in 
a mean gradient that is high, and that is why we are always worried about doing a valve-in-valve procedure. So if we were to compare and note that we prefer surgical AVR over transcatheter AVR in redo valve surgery, I think that we also should report the postoperative echocardiography findings. What are your thoughts and do you have any echocardiography data to share with us?

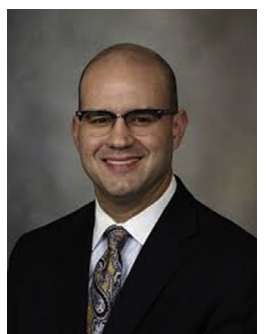

Dr John Stulak (Rochester, Minn). I don't have any immediate postoperative data. I know data from the valvein-valve international data (VIVID) registry showed that if there is baseline prosthetic aortic valve stenosis (compared with regurgitation) or a small valve size previously implanted, obviously those patients have a higher gradient. One would suppose in these patients that you would have a better postoperative gradient compared with transcatheter AVR valvein-valve procedure. I can't speak to that, but it would be intuitive to think that.

Dr Thourani. I can understand that doing a redo surgical AVR in a patient with a prior 19 or 21 valve. That's our practice currently unless it is a patient who really can't tolerate another surgery. But in those patients who have a 23 or a 25 and let's say 27 or 29 valves and who don't have permanent pacemaker, so that valve size is adequate for them, then it seems like, to me, a transcatheter AVR in appropriate high-risk patients in which they have the possibility to go home the next day and have a mean gradient under 10 , with no intubation nor intensive care unit stay is reasonable. I also think it depends on the age of the patient because we are still unsure of the thrombus rate in transcatheter AVR valve-in-valve patients.

So can you tell us from this study, because this is more of a generic study looking at it, what is the recommendation from the Mayo Clinic? If it's a 19 valve, are we saying do a redo surgical AVR, but if it's a 27 valve are you still saying regardless of age that we should be doing a redo surgical AVR or a transcatheter AVR valve-in-valve? How about an 82-year-old who has not had a previous coronary artery bypass grafting? Should we be doing redo AVRs in those patients or should we just go straight to transcatheter AVR?

Dr Stulak. It's a great question and not all my data is going to answer that. But just in looking at things, I think that a lot of us are tending to put in bigger valves, even if it means an aortic root enlargement in maybe the 60-year-old who wants a biological prosthesis. In an 82-year-old I would agree, with a good size valve probably you are not thinking of getting a 10-year survival out of that patient. However, in a 60 -year-old, even if they have a 23 or a 25 , even in the presence of a coronary bypass operation, I would be worried about doing a transcatheter AVR in a 60-year-old, because then you are looking at a redo transcatheter AVR at age 70 years, then maybe again at age 80 years.
As you said, the 80 -year-old patient with a 25 valve, that almost seems to be a no-brainer, again, the data weren't designed to answer that, but that would seem like the most logical step for that patient. A lot of it depends on patient condition, like you said, and really looking at it holistically.

Dr Thourani. Another group showed some good videos yesterday on root enlargements. I have been doing more root enlargements lately than I have ever done before, making sure that the patient gets a 23 or greater valve.

The last question I have for you is this: We still don't have great data on when is the time from implant to reoperation after a tissue AVR. Do you have any indication of the time from the original valve surgery to the time of the current redo valve surgery?

Dr Stulak. Yes. It's sporadic because a lot of patients had their first operation elsewhere and now they are coming to us for the redo. We've unfortunately had some people presenting less than a year later, so early degeneration. We've also had some people who I think the longest was 18 years for a pericardial valve. Although I can't say necessarily if there was a rhyme or reason, I saw the entire span of time frames. As we put in tissue valves in younger patients and not knowing the long-term durability of some of the newer-generation tissue valves, we may see that duration from the original implant to reoperation grow shorter, because we know the lifespan is shorter in younger patients. So that would be my general anecdotal response.

Dr Thourani. I see all my AVR patients back in the office for 30-day and 1-year echocardiography, and I would urge you that there is somewhere reported around a $10 \%$ to $15 \%$ rate of thrombus on these valves early, which I think may lead to early structural valve deterioration. So I think that we need to follow our own patients instead of just shipping them back to cardiologists and not seeing them forever again, because if you do see a higher gradient in 30 days, maybe you see them back early-at 3 months-where you do a 4-dimensional computed tomography scan to look at those. I urge the surgeons to do that.

I would like to congratulate you again, John, and your colleagues on an excellent study and an important contribution to the literature.

Dr Stulak. Thank you very much.

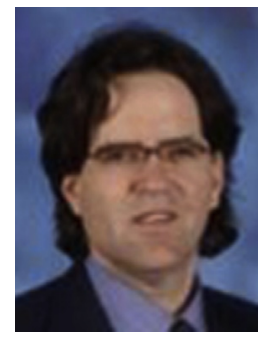

Dr Niv Ad (Falls Church, Va). Vinod, stay for a second. I have a comment. Did I hear you correctly that stented surgical valves have a higher rate of thrombosis than transcatheter AVR? You said $10 \%$ to $15 \%$ and the transcatheter AVR reporting is $13 \%$ or close to this.

Dr Thourani. It's all over the place. If you are doing 4-dimensional computed tomography scans on everybody, there are some reports of $30 \%$ of patients in a small series 
of surgical valves having thrombus. So right now I think the data are going to shake out somewhere between $5 \%$ and $20 \%$, but nobody knows until we do prospective 4-dimensional computed tomography scans following transcatheter AVR or surgical AVR. Currently all the low-risk surgical AVR/transcatheter AVR trials have a subcomponent of patients getting routine computed tomography scans after transcatheter AVRs and surgical AVRs. I think within the next 2 years we are going to have reliable, prospective data on exactly what that number is on hundreds of patients.

Dr Stulak. I think it's also incumbent to have a standardized approach to anticoagulation in these patients. Before some data coming out looking at that very presence of thrombus, we were not necessarily anticoagulating with warfarin. Now we do it for up to 6 months in some patients.

Dr Thourani. Yesterday in an aortic valve symposium, with a multitude of senior surgeons, we all noted that basically we have never thought about routine warfarin postoperatively, but based on a study out of Belgium, we are starting to think that maybe warfarin is something we should do for 6 months postimplant. As you know, we are in the midst of planning a potential randomized trial at the National Institutes of Health dealing with this exact topic.

Dr Ad. But Joe Sabik just showed earlier that their new valve has 0 thrombosis.

Dr Thourani. Correct me if I'm wrong, but they did not have 4-dimensional computed tomography scans. You can't diagnosis it just by saying I don't think you have it from a murmur. I think you have to evaluate it with a computed tomography scan. And an echocardiograph has been found not to be a very reliable test for that.

Dr Ad. I don't disagree with you. I just want to make sure that we all understand, we don't want to overtreat an 80-year-old with no gradient and no symptoms.

Dr Thourani. Absolutely, right.

Dr Ad. There is a difference between a small thrombus and no gradient and a larger thrombus with a gradient.
Dr Thourani. I 100\% agree with you. We need better prospective data on this.

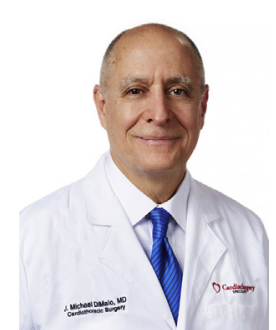

Dr J. Michael DiMaio (Dallas, Tex). We have a trial in Dallas where we have certain echocardiograph criteria and ejection fraction criteria and gradient criteria that were used to trigger the 4-dimensional computed tomography scans. I think you are right about the 4-dimensional computed tomography scans documenting the thrombus. Although it's not proven, I think some of that could be flow problems and we need to make sure that we are careful in what we consider the gold standard.

Dr Thourani. Absolutely.

Dr DiMaio. I know you know that but just to make sure the audience doesn't think that we are we not cognizant of the proper criteria for evaluation and documentation of possible thrombus.

Dr Thourani. Yes, you are right, absolutely.

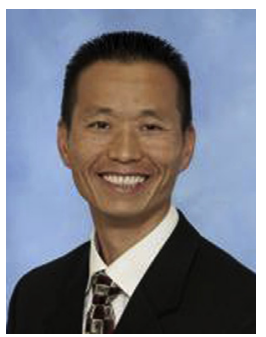

Dr Bo Yang (Ann Arbor, Mich). A great study. In your redo AVR, did you include any patient with a previous stentless valve, because a aortic valve reoperation for a previous stentless valve is more difficult, or more complicated, and what is the mortality in patients with redo AVR for a failed scentless valve? And because of the difficulty of the open reoperation for initial implanted scentless valve, do you think we should refer patients with failed stentless valve to transcatheter AVR more?

Dr Stulak. It's a good question. I can tell you that that was included because I have done some of those operations. I think the numbers are probably very small about explantation of the stentless valve. Other than the University of Michigan, and I don't want to speak for the other institutions, I know it's the preferred biological prosthesis there. I did not look at valve type, and I think the numbers would be very small to draw that conclusion. Sorry. 
TABLE E1. All candidate variables considered in the statistical models

Variable

Age

Aortic crossclamp time

Cardiopulmonary bypass time

Cerebrovascular disease

Chronic lung disease

Concomitant coronary artery bypass grafting

Diabetes

Hypertension

Immunosuppression

Left ventricular ejection fraction

Number of prior sternotomies

New York Heart Association functional class

Peripheral vascular disease

Prior coronary artery bypass grafting

Prior myocardial infarction

Redo aortic valve replacement

Redo mitral valve replacement

Renal insufficiency

Sex 